\title{
Promising Practices in Preconception Care for Women at Risk for Poor Health and Pregnancy Outcomes
}

\author{
Janis Biermann • Anne Lang Dunlop • Carol Brady • \\ Cynthia Dubin · Alfred Brann Jr.
}

Published online: 22 August 2006

(C) Springer Science+Business Media, Inc. 2006

\begin{abstract}
Objectives: Two programs targeting urban African-American women are presented as promising models for preconception care, which includes interconception care. Methods: The Grady Memorial Hospital Interpregnancy Care (IPC) Program in Atlanta, Georgia, and the Magnolia Project in Jacksonville, Florida, are described. The IPC program aims to investigate whether IPC can improve the health status, pregnancy planning and child spacing of women at risk of recurrent very low birthweight (VLBW). The Magnolia Project aims to reduce key risks in women of childbearing age, such as lack of family planning and repeat sexually transmitted diseases (STDs), through its case management activities. Results: Seven out of 21 women in the IPC were identified as having a previously unrecognized or poorly managed chronic disease. 21/21 women developed a reproductive plan for themselves, and none of the 21 women became pregnant within nine months following the birth of their VLBW baby. The Magnolia Project had a success rate of greater than $70 \%$ in resolving the key risks (lack of family planning, repeat STDs) among case management participants. The black to white infant mortality (IM) ratio was better for the babies born to women managed in the Magnolia Project compared to the same ration for the United
\end{abstract}

J. Biermann $(\bowtie)$

March of Dimes,

1275 Mamaroneck Avenue, White Plains, NY 10605, USA

e-mail: jbiermann@marchofdimes.com

A. L. Dunlop · C. Dubin · A. Brann Jr.

Emory University, School of Medicine,

1440 Clifton Road N.E., Atlanta, GA 30322, USA

C. Brady

Northeast Florida Healthy Start Coalition,

6850 Belfort Oaks Place, Jacksonville, FL 32216, USA
States. Conclusions: Preconception care targeted to AfricanAmerican women at risk for poor birth outcomes appears to be effective when specific risk factors are identified and interventions are appropriate. Outreach to women at risk and case management can be effective in optimizing the woman's health and subsequent reproductive health outcomes.

Keywords Preconception · Interconception · Birth outcomes $\cdot$ Risk reduction $\cdot$ Family planning

\section{Introduction}

Current national level statistics demonstrate the potential for preconception care to improve the health of women and impact their reproductive health outcomes in terms of achieving optimally spaced, planned pregnancies and averting adverse birth outcomes. Each year in the United States, approximately 6 million pregnancies result in over 4 million live births. Almost half of all pregnancies are unintended, despite the fact that $62 \%$ of the 62 million women of reproductive age use a contraceptive method [1]. Chronic diseases and adverse health behavior known to affect pregnancy outcomes are prevalent among women of reproductive age (1544 years). In 2002, 9.3\% of women of reproductive age had diabetes, and $11.4 \%$ of pregnant women smoked tobacco and $10.1 \%$ of pregnant women consumed alcohol [2].

The U.S. Public Health Service has designated preconception care as a critical component of prenatal care [3]. Initiating interventions to address a woman's health risks upon her entry into prenatal care is often too late to impact the outcome of pregnancy. For example, if a woman has her first prenatal visit after the first month of pregnancy, it is too late to prescribe folic acid supplementation to help prevent neural tube defects as the neural tube closes by the 28th day 
of gestation. Likewise, women who are advised to discontinue their use of potentially teratogenic medications at the first prenatal visit may have already sustained damage to the fetus, as most organogenesis is complete by day 56 following conception [4].

Preconception care is the care of women of reproductive age before a first pregnancy or between pregnancies (commonly known as interconception care) to ensure that conditions and behaviors which may pose a risk to mothers and infants are identified and managed. A specific element of interconception care is the identification and reduction of risks indicated by a prior adverse pregnancy outcome. The major components of preconception care are risk assessment (identification of conditions that may be amenable to intervention that adversely affect reproductive health outcomes), education and health promotion, and medical and psychosocial interventions.

There are varying concepts of preconception care ranging from a pre-pregnancy planning visit in the months prior to conception to a component of each health encounter for women. Ideally, preconception care would be an integral component of primary and preventive care for women of reproductive age. Presently, the status of preconception care in the United States is far from ideal. Approximately one of six obstetrician/gynecologists or family physicians had provided preconception care to the majority of the women for whom they provided prenatal care [5].

Given the diversity of women of reproductive age, the numerous health and psychosocial issues that impact reproductive health outcomes and the varied settings for delivery of health care, a single 'best' model of preconception care for all women is unlikely. Rather, tailoring the delivery of preconception care interventions to women with specific circumstances and in specific care settings will be necessary. This paper describes two programs for delivering preconception care for women at-risk for poor health and pregnancy outcomes. One targets women whose risk status is defined by African-American ethnicity and delivery of a very low birth weight (VLBW; $<1500 \mathrm{~g}$ ) infant and, thus, specifically involves the provision of an interconception intervention. The other program targets women whose risk status is defined by African-American ethnicity and residence in JacksonvilleDuvall County, Florida, and involves the delivery of an intervention with preconception and interconception components.

\section{The grady memorial hospital Interpregnancy Care (IPC) program}

\section{Background}

Georgia ranks among the 10 states with the highest fetoinfant mortality rate. The largest contributor to Georgia's comparatively high feto-infant mortality is the delivery of VLBW infants. VLBW delivery comprises only $2 \%$ of births in Georgia, yet accounts for approximately $50 \%$ of fetoinfant mortality. African-American women in Georgia have 3-4 times the rate of VLBW delivery compared to whites. Approximately $66 \%$ of the observed racial disparity in fetoinfant mortality rates between African-Americans and whites can be attributed to the higher rate of VLBW delivery among African-American women [6].

There is a close link between the poor health status of women and VLBW delivery [7-17]. The poor health status of women in Georgia is estimated to account for approximately $60 \%$ of Georgia's excessive infant mortality [18]. With each subsequent VLBW delivery, there is an increased chance that the woman's next pregnancy will result in recurrence of VLBW delivery [19]. In fact, the best predictor of whether a woman will have a VLBW delivery is her history of a previous VLBW delivery, with the rates of recurrence after a single VLBW delivery being approximately 1.5 times higher for African-American compared to white women (13.4\% vs. $8.2 \%$, respectively, for non-teen mothers; $26.8 \%$ vs. $16.4 \%$, respectively, for teen mothers). The reason for recurrence of VLBW delivery is likely that aspects of the woman's pre-existing health status-including untreated or poorly managed medical problems and unaddressed nutritional, social, and behavioral risk factors-that may have contributed to delivery of the VLBW delivery persist after delivery and in subsequent pregnancies.

In 1998 the Georgia Task Force on Perinatal Care was convened to make recommendations for reducing Georgia's overall feto-infant mortality rate and racial disparities in fetoinfant mortality. From its findings as outlined above, the Task Force specifically recommended that interpregnancy care be initiated and evaluated for women at risk for having recurrence of VLBW delivery. The Grady Memorial Hospital Interpregnancy Care (IPC) Program was initiated in response to the recommendation of the Task Force.

\section{Target population}

African-American women residing in Fulton or DeKalb counties, Georgia who qualify for county-supported indigent care services and who deliver liveborn or stillborn VLBW infants at Grady Memorial Hospital in Atlanta are considered eligible for the IPC program.

\section{Program description}

The Grady Memorial Hospital IPC program provides 24 months of integrated primary health care and dental services through enhanced nurse case management and community outreach via a Resource Mother. The Resource Mother 
is a layperson who is trained by the Fulton County Health Department using a specific set of objectives to facilitate life skills and health education acquisition to support high-risk women. The nurse case manager offers all women who deliver a VLBW (stillborn or liveborn) infant at Grady Memorial Hospital enrollment in the program during or soon after their delivery admission. Women who choose to enroll in the IPC program have their initial home visit with the Resource Mother within 1-2 weeks of discharge from the delivery admission; they are scheduled for their initial IPC clinical evaluation at a clinic within Grady Memorial Hospital at 4-6 weeks postpartum.

At the initial IPC clinical evaluation, the IPC program's family physician or nurse- midwife performs a comprehensive survey of medical, obstetrical, nutritional, psychological, and social issues (using standardized assessment tools); a thorough physical examination including pelvic exam; and laboratory evaluations to screen for anemia, nutritional deficiencies, sexually transmitted diseases (STDs) and reproductive tract infections. Standard postpartum care issues are addressed in the initial IPC clinical evaluation, thus eliminating a separate postpartum appointment.

As part of the evaluation, the participant and provider explicitly discuss a care plan for the 24-month period of the program. For each woman, the care plan addresses the following seven areas epidemiologically linked to LBW delivery: 1) Pregnancy intendedness and child-spacing through the provision of health education concerning the importance of achieving at least a 9-month (and preferably an 18-month) interpregnancy interval, assisting the woman to articulate her own reproductive plan and select a corresponding contraceptive method; 2) Management of chronic disease (if present) through the promotion of self-care and adherence to scheduled appointments in Grady Health System that are facilitated by nurse case management; 3) Screening and treatment for nutritional deficiencies; 4) Prevention, screening, and treatment for STDs and reproductive tract infections; 5) Treatment and referral for substance abuse (if present) including linkage with rehabilitation programs for illicit substance abuse, and support in and linkage with existing programs for tobacco and alcohol abuse; 6) Screening and treatment or support for depression, psychosocial stressors, and domestic violence; 7) Prevention, screening and treatment for periodontal disease.

Subsequent visits to the IPC clinic are offered every 13 months, depending upon the extent and severity of the woman's health and social issues. Elements of the care plan are addressed at each subsequent visit to the IPC clinic. Peer group learning experiences are integrated with IPC health care visits and are modeled on the Centering Pregnancy concept of group prenatal care [20].

Resource Mother support services are focused on identification and management of psychosocial stressors, and life skills enhancement, including parenthood preparedness, safe housing, skills training, employment acquisition, and relationship issues. Resource Mother support is offered at least twice monthly in the form of home visits and telephone contact. Participants are able to contact the Resource Mother via her cell phone to request support, if needed.

Providers

Primary care and outreach services are delivered by a multidisciplinary team comprised of a family physician, an advanced practice nurse trained as both a nurse midwife and a family nurse practitioner, a periodontist, a nurse case manager, and a Resource Mother. Social workers affiliated with Grady Health System also support the IPC program and interface with the IPC team.

Outcome objectives

The purpose of the Grady Memorial Hospital IPC program is to investigate whether interpregnancy care can improve the health status, pregnancy planning and child spacing of women at risk of recurrent VLBW delivery. Findings will contribute to the field of primary health care of reproductive age women in several important ways: (a) the content of a successful IPC package for improving the health of high-risk women will be explored; (b) the concept of IPC will be tested as a means of improving attainment of desirable interpregnancy intervals and decreasing the occurrence of subsequent adverse pregnancy outcomes for high-risk women; (c) the cost of providing IPC to high-risk women will be studied.

\section{Funding}

The health care services rendered to IPC participants were provided through the services of the Grady Health System. Funding for the research and evaluation components of the IPC Program, including Vasser-Wooley Foundation, Healthcare Georgia Foundation, Centers for Disease Control and Prevention (CDC), Rockdale Foundation, and March of Dimes.

Results from the first year of the IPC program

\section{Enrollment}

During November 2003 through March 2004, the feasibility phase of the IPC program was initiated by enrolling the first sequential 29 eligible women who gave their informed consent for participation. During the enrollment period, a total of 47 women delivered VLBW infants at Grady Memorial Hospital. Of these 47 women, nine were not AfricanAmerican or did not reside in Fulton or DeKalb counties, 
four declined enrollment, three who wished to be contacted afterdischarge from the hospital were unable to be contacted, and two were discharged from the hospital within $24 \mathrm{~h}$ and before being offered enrollment.

\section{Participation}

Twenty-one of 29 enrolled women (72.4\%) successfully completed the initial 12 months of the planned 24 months of follow-up. During the first 12 months, eight women became disenrolled from the program two moved out of state (and informed us of their move), three electively disenrolled (two before prior to the initial home visit and the initial IPC clinical evaluation; one after a single IPC clinic visit), and three became lost to follow-up (two before the initial IPC clinical evaluation; one after a single IPC clinic visit and four home visits) all of whom who had noted problems with cocaine abuse in their medical records during their pregnancy.

\section{Outcomes}

A detailed program description and final results for all evaluated outcomes for the full 24 months of follow-up will be published when all follow-up and outcome ascertainment is complete (anticipated June 2006). Important findings related to the health status of women in the IPC intervention cohort who had at least two visits during the first 12 months of IPC are given below:

- Chronic diseases that were previously unrecognized or poorly managed were identified for $7 / 21$ participants, and include valvular heart disease (1/21), sickle cell anemia $(1 / 21)$, hypertension $(2 / 21)$, diabetes $(1 / 21)$, asthma $(1 / 21)$, systemic lupus erythematosus $(1 / 21)$, prolactinoma $(1 / 21)$, panic attacks (1/21), and generalized anxiety disorder $(1 / 21)$;

- Reproductive tract infections were diagnosed and treated for 15/21 participants;

- Iron-deficiency anemia was diagnosed and treated for $5 / 21$ participants;

- Concerns about finances, employment, and needs of the child are almost universal stressors;

- Average annual outpatient charges per participant for IPC are $\$ 1,801$ (average 4.6 visits, $\$ 389$ per visit).

Important findings related to the reproductive outcomes during the first 12 months of IPC are as follows:

- With extensive case management and patient education, 21/21 women who had at least two visits during their first 12 months of IPC, stated a reproductive plan for themselves and initiated a contraceptive plan in accordance with their stated reproductive plan. Despite a stated desire to either avoid or delay a pregnancy (21/21 women), a sig- nificant number of barriers to effective contraception existed and were dealt with, including misinformation about contraceptive methods and reproduction, concerns about side effects of contraceptive methods, and perceptions of partners' desires regarding child bearing and contraception.

- All of the women who had at least two visits during their first 12 months of IPC (21/21) achieved at least a ninemonth interpregnancy interval (i.e., none have become pregnancy within nine months of the index VLBW delivery). For the 21 actively participating women, pregnancy ascertainment involved ongoing contact with the women and reviewing the Grady Healthy System medical record system for any evidence of health care visits or laboratory tests in which a pregnancy was diagnosed, incidentally noted, or treated. No pregnancies are known to have occurred within nine months of the index VLBW delivery for the eight women who became disenrolled, for which pregnancy ascertainment involved telephone follow-up, where possible (two women), and review of the Grady Health System medical record system. It is possible that those women with whom contact was lost have had a pregnancy for which no care was sought or care was sought outside of Grady Health System.

\section{Barriers}

In the United States, a major barrier to studying the impact of interpregnancy care on the health status and reproductive outcomes of high-risk women has been the lack of financial coverage for the health care component of the IPC intervention. For the IPC program in Atlanta, the Grady Health System has provided the two years of primary health care and treatment of all identified diseases for all enrollees.

Specific barriers exist for retaining participation in the IPC program. Women with substance abuse problems who do not enroll in formal substance abuse treatment programs are difficult to maintain in the IPC program. These women seem to have poor insight into the effects that substance abuse has on their repeated poor pregnancy outcomes. For some enrolled women, receiving health care services is less of a priority than securing employment, which negatively influences their health care seeking behaviors.

\section{Next steps}

Upon conclusion of the planned 24 months of follow-up, the final results for all evaluated outcome objectives from the feasibility phase will be reported. The IPC program is currently seeking grant support to conduct a randomized clinical trial to test the hypothesis of whether IPC can decrease the occurrence of subsequent adverse pregnancy outcomes for pregnancies conceived within 18 months of a VLBW 
delivery at Grady Memorial Hospital. The project plans to enhance participant retention by offering more opportunities for life skills enhancement, job training, and delivery of health care services via the community-based Grady Neighborhood Health Centers.

\section{The magnolia project}

\section{Background}

In 1998, infant mortality rates in Jacksonville-Duval County, Florida were increasing while statewide rates were declining. The Northeast Florida Healthy Start Coalition, Duval County Health Department and other community partners undertook a community assessment and identified racial disparities as a major factor contributing to the city's high infant mortality. The Coalition utilized the Perinatal Periods of Risk (PPOR) and findings from its Fetal \& Infant Mortality Review (FIMR) project to analyze linked birth and death data. The results of this analysis indicated the need for interventions that focused on the health of women, particularly African-American women, before conception. The Coalition used this information to apply for federal Healthy Start funding under a 1999 initiative to address racial disparities in birth outcomes. This funding was used to establish The Magnolia Project, which adapts selected Healthy Start program models to at-risk African-American women of childbearing age who are not pregnant, but sexually active and likely to become pregnant. The project is implemented in a five-zipcode area of Jacksonville-Duval County that accounts for over half of all African-American infant mortality and about $30 \%$ of African-American births.

\section{Target population}

The Magnolia Project targets African-American women ages 15-44 living in a socioeconomic high-risk area of Jacksonville-Duval County. The project focuses on women of childbearing age who are likely to become pregnant and have some identified risk factor associated with poor birth outcomes. The project also maintains a small caseload of pregnant women $(<10 \%$ of patients).

Program description

The Magnolia Project provides outreach, case management, risk reduction, support, well-woman prenatal care, health education, and community development. The project engages high-risk women through an empowerment model that promotes improved wellness and health, rather than future childbearing. Clinical services and health education are available to all eligible women in the target area. The project offers intensive case management to a subset of women who have specific risk factors identified through FIMR, including previous fetal or infant death or delivery of a LBW infant; repeated STDs; lack of family planning; substance abuse; first pregnancy before age 15; and lack of access to health care.

The project's intensive case management is a collaborative team approach that supports participants based on a comprehensive assessment and a client care and goal plan. The plan is developed in collaboration with each participant and reflects her choices, preferences, and goals for coordination of services and activities that address identified risk factors associated with infant mortality. Case management services include:

- A comprehensive assessment of each participant's abilities and needs at program entry. This assessment includes completion of a problem checklist; screening for depression and stress; and evaluation of self-esteem. Participants are re-assessed periodically based on need and progress to determine resolution or recurrence of problems and risks, achievement of personal goals, and improvements in coping skills and self-esteem.

- Participant care and goal plan. This plan addresses the needs and preferences of each participant. The written plan is a collaborative process involving the participant and the case manager. It addresses health and social risks and outlines specific steps that the participant must follow to improve her health and well-being and to meet her goals for social, economic, and personal development.

- Ongoing monitoring and service coordination. The case management team monitors services to ensure that quality care is being provided, participants are addressing their care plans, and participants who are referred to another agency follow through and receive appropriate services. The risk profile (problem checklist) and other measures (stress, self-esteem) are updated at regular intervals (at 6 and 9 months).

- Anticipatory guidance, health education and advocacy. Case management staff addresses participant risk factors, ensures compliance with services, and promotes achievement of personal goals. Educational support groups are organized monthly. Women receive an incentive ( $\$ 50$ gift certificate) for each six months that they remain in the program, provided they have made progress in addressing their goals.

\section{Providers}

The clinical component of the program is staffed by a nursemidwife and related nursing, lab and support staff. The program has a full-time health educator who provides a brief counseling intervention on key issues (e.g., use of multivitamins, nutrition, douching, and safe sex) to every woman 
who comes into the clinic. The clinic serves approximately 800 women annually. A team that includes a nurse, a social worker and two specially trained paraprofessionals provides intensive case management to about 75 women a year. Community development and outreach staff, as well as members of the Magnolia Project Community Council, who are indigenous neighborhood leaders, conduct outreach and community awareness activities. The project uses a collaborative, multi-agency staffing model. The Northeast Florida Healthy Start Coalition is the grantee and project administrator. Subcontractors include the Duval County Health Department (clinical care and case management) and Shands Jacksonville, a tertiary care and teaching hospital (case management and outreach). Staff is co-located at a storefront community site within walking distance of six public housing complexes. The University of North Florida Center for Community Initiatives serves as project evaluator.

\section{Outcome objectives}

The project addresses all the outcome objectives required by the Maternal and Child Health Bureau of the Health Resources and Services Administration (HRSA) regarding birth outcomes (LBW, VLBW, infant mortality, adequacy of prenatal care, etc.). However, because the project primarily focuses on women who are not pregnant, it has developed specific outcome objectives that examine its success in reducing key risks associated with poor outcomes through its case management activities. These include lack of family planning and STDs. Specific outcome objectives have been developed to measure the program's success in addressing participant risks identified at program entry and closure.

\section{Funding}

The Magnolia Project is primarily funded through a grant from the federal Healthy Start program $(\$ 925,000)$. Additional funding is provided through Medicaid and other thirdparty reimbursement for prenatal, family planning and STD services; state categorical funding through the health department (about $\$ 250,000$ a year); and smaller community grants for special projects. Total 2004-2005 budget was just over $\$ 1$ million.

Results to date

From 2001 to 2005, Magnolia Project clients were predominantly African-American (83\%) and single (82\%). The average age of participants was 25 years old. More than half $(55 \%)$ had less than a high school education. Based on patient history and assessment, case management participants had an average of 7.9 risk factors or problems (current or history of) that could potentially impact a pregnancy. Seventy-two
Table 1 Frequency of risks/problems, case management participants, The Magnolia Project, July 2001-May 2005

\begin{tabular}{lcc}
\hline & $\begin{array}{l}\text { Percentage with } \\
\text { history } \\
(n=1214)\end{array}$ & $\begin{array}{l}\text { Percentage with } \\
\text { current } \\
(n=1727)\end{array}$ \\
Risk factor/problem & & \\
\hline Social problems & 26 & 43.5 \\
Family planning issues & 8.2 & 33.0 \\
Job placement & 11.3 & 27.3 \\
Education/training & 8.7 & 21.7 \\
Stress & 4.1 & 15.7 \\
Housing & 7.2 & 11.3 \\
Domestic violence & 8.2 & 11.1 \\
Depression & 6.7 & 8.7 \\
No source of care & 7.0 & 8.7 \\
Lack of exercise & 2.8 & 8.0 \\
Injury prevention/safety & 10.0 & 6.7 \\
Drug abuse & 4.4 & 5.9 \\
Transportation & 7.0 & 4.4 \\
Sexual abuse & 6.4 & 4.1 \\
Alcohol abuse & & \\
Medical problems & 12.9 & 30.2 \\
Bacterial vaginosis & 17.8 & 19.3 \\
Poor nutrition & 12.4 & 16.5 \\
Repeated STDs & 13.2 & 15.5 \\
Tobacco use & 3.9 & 11.3 \\
Breast health & 8.5 & 10.8 \\
Douching & 9.5 & 9.5 \\
Abnormal pap & 11.6 & \\
Overweight & & \\
\hline
\end{tabular}

Source: Magnolia Project database; compiled by UNF Center for Community Initiatives, August 2005.

percent of all current problems were social problems (alcohol use, drug abuse, depression, family planning, housing, stress, and domestic violence). The remainder were medical problems (anemia, diabetes, hypertension, poor nutrition, previous poor outcome, STDs). The most common risk factors addressed in case management were family planning issues, education/training, bacterial vaginosis, repeated STDs, stress, and poor nutrition. Table 1 identifies frequency of participant risks/problems.

Two-hundred-forty-seven participants received at least three months of case management and were closed to care because they completed their care plans, voluntarily withdrew or were lost to service. For key risk factors, case management participants were most successful in resolving or managing issues related to domestic violence $(68 \%)$ and poor nutrition $(63 \%)$. The project was least successful in addressing substance abuse by participants (31\%).

In October 2004, the HRSA Office of Performance Review conducted a site visit and assessment of Magnolia Project outcomes and activities. Its report noted that participants receiving case management services from 2001 to 2003 successfully addressed two priority risks: $86 \%$ 
of participants with family planning issues were consistently using a method at closure; $74 \%$ of participants with repeated STDs had no recurrent STDs at closure.

\section{Barriers}

Insufficient funding has limited expansion of the Magnolia Project. Existing funding streams focus primarily on pregnancy, not women's health. The Magnolia Project has successfully cobbled together categorical funding and Medicaid reimbursement for most clinical services, however, in Duval county, the delivery of most categorically funded services remains siloed (e.g., STDs, primary care, prenatal care).

Additionally, case management/risk assessment is not universally accepted or valued in the medical model of health care, even though many of the risk factors associated with poor outcomes are social, rather than medical, in nature. More longitudinal evaluations are needed to determine the impact of preconception intervention on pregnancy outcomes. Longitudinal evaluations are not easily incorporated into service delivery projects that rely on short-term outcomes for continued funding. Finally, although outcomes have improved in the target area since the implementation of the Magnolia Project, infant mortality rates in the overall community remain high. The project is unlikely to affect these rates because of its comparatively small reach.

\section{Next steps}

The Magnolia Project is aggressively seeking funding for a longitudinal evaluation. Current program evaluation is limited to an assessment of how well the project is achieving short-term objectives and objectives set by the funding agency (primarily focusing on birth outcomes for the small number of pregnant women served by the project). The CDC recently provided support to the Duval County Health Department for the initial design and piloting of tools for a longitudinal assessment of case management participants served by the project. Additionally, the project is working to interest local providers in replicating the intervention in other at-risk areas in Jacksonville-Duval County. Funding for replication is a significant challenge.

\section{Discussion}

The Grady IPC program and the Magnolia project use a classic public health model of care. They identify women at known risk for an adverse outcome and attempt to alter the woman's risk status to improve outcomes. These programs deliver aspects of preconception care to women at risk for poor health and pregnancy outcomes. The programs do, however, target different categories of high-risk African-American women and utilize different sites for con- tacting and interfacing with them. The IPC program identified women at risk based on race/ethnicity, qualification for charity care (based on financial status and geographic residence in two counties of metropolitan Atlanta) through Grady Memorial Hospital, and a prior poor birth outcome (VLBW delivery). The IPC program enrolled women soon after their VLBW delivery and provided interconception care in the clinical setting of the hospital (with community outreach). The Magnolia project serves women at risk based on race/ethnicity and residence in Jacksonville-Duval County, Florida. It provides preconception care in a communitybased storefront setting with enhanced interconception care for women with a previous LBW delivery or a previous fetal or infant death. Access to the Magnolia project is enhanced by offering evening clinics and walk-in Wednesdays.

Both programs deliver services via a multidisciplinary team approach, though there are differences in the exact composition of the team. Inherent in the team approach of each program is attention to participants' physical and psychological health and social well-being.

The programs emphasize several comparable intervention strategies, including community outreach via lay persons; psychosocial support; group education and health promotion modeled on the Centering Pregnancy philosophy of care; and provision of health services. Both programs also emphasize the provision of family planning services for helping women achieve intendedness of any subsequently conceived pregnancy.

Each program offers unique strategies as part of the interconception intervention. Because of the link between periodontal disease and preterm delivery (almost all VLBW deliveries are preterm), the IPC program incorporates the services of a periodontist to screen and treat women. To specifically enhance particpants' life skills, the IPC program utilizes a layperson as a Resource Mother who has undergone a tailored training program through the local department of health. A unique feature of the Magnolia Project's approach is its reliance on indigenous neighborhood leaders (e.g., Magnolia Project Community Council) and community-based organizations to provide outreach, community awareness, and education. The Magnolia Project also has established a partnership with the Ryan White III program for STD/HIV/AIDS screening and treatment.

Both programs have had success in accessing and providing services to the specific target group of women. Women who choose not to avail themselves of the services seem to have greater socioeconomic issues, such as substance abuse and lack of housing, jobs, and childcare. Due to the magnitude of some of the problems, maintaining contact is challenging and care often is not continuous.

Existing literature supports the strategy of addressing family planning to improve pregnancy outcomes. Preterm delivery, the underlying factor responsible for most LBW and 
VLBW deliveries, is the leading cause of neonatal mortality and among the leading causes of infant mortality. Haas and colleagues examined whether a woman's health status and risk factors before pregnancy are associated with her risk of preterm birth, independent of risk factors that occur during pregnancy. They found that, after adjusting for sociodemographic characteristics and prepregnancy and pregnancy risk factors, women who reported poor physical function during the month before conception were nearly twice as likely to deliver preterm. The authors concluded that a broader focus on the health of women before pregnancy might improve rates of preterm birth [21].

In a related editorial, Goldenberg suggests that reducing smoking, depression, and stress; treating periodontal disease and genital tract infections; and reaching a normal weight might all be necessary to substantially reduce the risk of preterm birth [22]. Goldenberg advocates for a Medicaid mandate that women who have had an adverse pregnancy outcome be eligible for continued medical coverage in the postpartum period to reduce risk factors and improve health status before the next pregnancy.

Data from the 1999 PRAMS survey indicate that the prevalence of unintended pregnancies in the United States ranges from 34 to $52 \%$ with 27 to $36 \%$ of these pregnancies being mistimed, and 6 to14\% being unwanted [23]. In the United States, a clear causal association between unintended pregnancy and poor pregnancy outcomes has not been established. However, women with unwanted pregnancies are at greater risk for poor pregnancy outcomes than women with wanted pregnancies. Hogue asserts that one of the key approaches to prevention of high-risk pregnancy is assuring that the pregnancy is consciously desired, with adequate attention to preconception care [24].

Both programs demonstrate several components essential to any successful preconception care program: identification of risk factors amenable to change by the target population; tailored interventions for the target population; integration of preconception care into existing services; incorporation of family planning counseling and clinical services, health education, and community outreach. Preconception care aims to promote the health of women of reproductive age before conception and thereby improve pregnancy-related outcomes. Both the IPC program and the Magnolia Project show promise in achieving this goal for their target audiences.

\section{References}

1. The Alan Guttmacher Institute. Facts in brief: Contraceptive use. Available: http://www.agi-usa.org/pubs/fb_contr_use.html; September, 2005.

2. US Department of Health and Human Services, Health Resources and Services Administration, Maternal and Child Health Bureau. Women's Health USA 2002. Rockville, MD: US Department of Health and Human Services.
3. US Public Health Service, Expert Panel on Health and Human Services. Caring For Our Future: The Content of Prenatal Care: A Report of the Public Health Service Expert Panel on the Content of Prenatal Care. Washington, DC: Public Health Service, US Dept. of Health and Human Services, 1989.

4. Moore K, Persaud TVN. Developing human. Philadelphia: WB Saunders; 1998.

5. Henderson JT, Weisman CS, Grason H. Are two doctors better than one? Women's physician use and appropriate care. Women's Health Issues 2002;12:139-49.

6. Iyasu S, Becerra JE, Rowley DL, et al. Impact of very low birthweight on the black-white infant mortality gap. Am J Prev Med 1992;8:271-7.

7. Kovilam O, Khoury J, Miodovnik M, et al. Spontaneous preterm delivery in the type 1 diabetic pregnancy: The role of glycemic control. J Mat-Fetal \& Neonatal Med 2002;11:245-8.

8. Sibai B, Caritis SN, Hauth JC, et al. Preterm delivery in women with pregestational diabetes mellitus or chronic hypertension relative to women with uncomplicated pregnancies. Am J Obstet Gynecol 2000;183:1520-4.

9. Lockwood CJ. Heritable coagulopathies in pregnancy. Obstet Gynecol Surv 1999;54:754-9.

10. Jacobsson B, Pernevi P, Chidekel L, et al. Bacterial vaginosis in early pregnancy may predispose for preterm birth and postpartum endometritis. Acta Obstetricia et Gynecologica Scandinavica 2002;81:1006-10.

11. Offenbacher S, Lieff S, Boggess KA, et al. Maternal periodontitis and prematurity. Part I: Obstetric outcome of prematurity and growth restriction. Ann Periodontol 2001;6:164-74.

12. Madianos PN, Lieff S, Murtha AP, et al. Maternal periodontitis and prematurity. Part II: Maternal infection and fetal exposure. Ann Periodontol 2001;6:175-82.

13. Cnattingius S, Bergstrom R, Lipworth L, et al. Pre-pregnancy weight and the risk of adverse pregnancy outcomes. N Engl J Med 1998;338:147-52.

14. Sebirea NJ, Jollya M, Harris J, et al. Is maternal underweight really a risk factor for adverse pregnancy outcome? A population-based study in London. BJOG: An Int J Obstet Gynaecol 2001;108:61-6.

15. Xiong $X$, Buekens $P$, Alexander $S$, et al. Anemia during pregnancy and birth outcome: A meta-analysis. Am J Perinatol 2000;17:13746.

16. Scanlon KS, Yip R, Schieve LA, et al. High and low hemoglobin levels during pregnancy: Differential risks for preterm birth and small for gestational age. Obstet Gynecol 2000;96:741-8.

17. Kelly RH, Russo J, Holt VL, et al. Psychiatric and substance use disorders as risk factors for low birth weight and preterm delivery. Obstet Gynecol 2002;100:297-304.

18. Georgia Perinatal Task Force. Report of the Task Force on the Georgia Perinatal Program. Atlanta, GA: Georgia Perinatal Task Force, 1998.

19. Adams MM, Elam-Evans LD, Wilson HG, et al. Rates of and factors associated with recurrence of preterm delivery. JAMA 2000;283:1591-6.

20. Rising SS. Centering pregnancy. An interdisciplinary model of empowerment. J Nurse-Midwifery 1998;43(1):46-54.

21. Haas JS, Fuentes-Afflick E, Stewart AL, et al. Prepregnancy health status and the risk of preterm delivery. Arch Pediatr Adolesc Med 2005; 159:58-63.

22. Goldenberg RL, Culhane JF. Prepregnancy heath status and the risk of preterm delivery. Arch Pediatr Adolesc Med 2005;159:89-90.

23. US Department of Health and Human Services, Centers for Disease Control and Prevention: Pregnancy Risk Assessment Monitoring System (PRAMS): PRAMS and unintended pregnancy. Available: http://www.cdc.gov/prams/up.htm. October 2005.

24. Hogue CJ, Vasquez C. Toward a strategic approach for reducing disparities in infant mortality, rural health and women of color. Am J Public Health 2002;92(4):552-5. 